\title{
Red Blood Cell Aggregation in Preterm and Term Neonates and Adults
}

\author{
OTWIN LINDERKAMP, PATRICK OZANNE, PAUL Y. K. WU, AND HERBERT J. MEISELMAN \\ Department of Pediatrics, University of Heidelberg, Federal Republic of Germany and Department of Physiology \\ and Biophysics and Department of Pediatrics, University of Southern California, Los Angeles, California 90024
}

\begin{abstract}
Aggregation of red blood cells (RBC) is a major determinant of blood viscosity and of blood circulation through vessels with slow flow (i.e. veins). RBC aggregation and plasma fibrinogen were studied in placental blood samples from 25 neonates with 24 to 41 wk of gestation and in blood from 13 normal adults. The rate and final extent of RBC aggregation were measured by means of a rheoscope (increase in light transmission during blood stasis). Both the rate and extent of RBC aggregation were low in the premature infants, increased with gestational age, and reached the highest values in the adults. Blood from seven infants with 24 to 28 wk of gestation did not show any significant $\mathrm{RBC}$ aggregation during the first minute of stasis. $\mathrm{RBC}$ aggregation was closely related to the fibrinogen level. Cross-suspension studies (neonatal $\mathbf{R B C}$ in adult plasma and adult $\mathrm{RBC}$ in neonatal plasma) showed that neonatal and adult $\mathrm{RBC}$ had the same aggregation pattern when they were suspended in the same plasma. Moreover, neonatal and adult RBC demonstrated the same strong aggregation when they were resuspended in $1 \%$ dextran. These results indicate that specific plasma properties are responsible for the decreased RBC aggregation observed in the neonates while their specific RBC properties do not affect RBC aggregation. (Pediatr Res 18:1356-1360, 1984)
\end{abstract}

\section{Abbreviations}

RBC, red blood cell(s)

AHT, aggregation half-time

RLT, relative light transmission

GA, gestational age

BW, birth weight

PBS, phosphate-buffered saline

Aggregation of $\mathrm{RBC}$ is a physiological property of blood which causes RBC to be arranged in rouleaux (22). The following processes are involved in the formation of RBC aggregates: 1) interaction of $\mathrm{RBC}$ and membrane deformation to increase the contact area; 2) formation of bridges between adjacent RBC by macromolecules such as fibrinogen, immunoglobulins, or high molecular weight dextran; 3) formation of a three-dimensional network of rouleaux (Fig. 1). These processes require several seconds of contact between adjacent RBC and, therefore, occur

Received November 30, 1983; accepted June 18, 1984.

Requests for reprints should be addressed to O. Linderkamp, M.D., UniversitätsKinderklinik Heidelberg, Im Neuenheimer Feld 150, D-6900 Heidelberg 1, Federal Republic of Germany.

This work was supported by the Deutsche Forschungsgemeinschaft Grant Li 291/2-2 (for research in the U. S. A.) and National Institutes of Health Grants HL 15722 and HL 15162 . only during stasis or at low shear stresses $(5,22,23)$. At high shear stresses (about 3 dynes $/ \mathrm{cm}^{2}$ or more), RBC aggregates are rapidly dispersed (23). Thus, RBC aggregation usually takes place only in the venous portion of the circulation where the blood flow rate is slow and the fluid shear stresses are low. RBC aggregation may occur in other sections of the circulation, however, under pathophysiological situations of reduced blood flow (i.e. shock). Conversely, increased $\mathrm{RBC}$ aggregation (e.g. as a result of high fibrinogen level) can cause lower blood flow rates, and this latter mechanism has been suggested as a possible factor in the pathogenesis of circulatory and thromboembolic complications in septicemia, pregnancy, diabetes, etc. $(18,20,22)$.

In term neonates, the rate and the final extent of RBC aggregation are lower than in adults $(7,8,24)$. No information is available on $\mathrm{RBC}$ aggregation in preterm infants. In addition, it is unknown whether specific properties of neonatal RBC contribute to the decrease of aggregation. In this context, it is interesting to note that specific cellular factors are responsible for the impaired aggregability of both platelets and polymorphonuclear leukocytes in the neonate $(9,19)$.

The present study was designed to measure the dynamics and the final extent of (in vitro) RBC aggregation of neonates with gestational ages of 24 to 41 weeks. Furthermore, we determined the isolated influence of plasma and of RBC on the aggregation process by suspending $\mathrm{RBC}$ from one adult donor in the plasma samples of the neonates and by suspending the RBC from the neonates in an aggregating dextran solution and in plasma from adults.

\section{MATERIALS AND METHODS}

Placental blood samples from seven preterm infants with GA of 24 to $28 \mathrm{wk}$ and BW of 690 to $990 \mathrm{~g}$, eight preterm infants with GA of 29 to $34 \mathrm{wk}$ and BW of 1080 to $2140 \mathrm{~g}$, and 10 healthy full term neonates with GA of 38 to 41 wk and BW of 2900 to $3570 \mathrm{~g}$ were studied with the approval of the University of Southern California Human Subjects Research Committee. Infants with malformations, erythroblastosis, diabetic mothers, hemorrhage, and intra-uterine asphyxia, and those delivered by cesarean section were excluded, as were twins and infants with a high risk of infection. All infants had birth weights less than the 90 th and more than the 10th percentile for gestational age.

In the 25 neonates, $10 \mathrm{ml}$ of placental blood were collected from the cord into heparin $(5 \mathrm{IU} / \mathrm{ml})$ immediately after cord severage prior to delivery of the placenta. Adult blood samples were collected from 13 healthy laboratory personnel via venipuncture into heparin. All measurements were made at room temperature $\left(22 \pm 1^{\circ} \mathrm{C}\right)$ within $4 \mathrm{hr}$ after collection. $\mathrm{RBC}$ were isolated by centrifugation at $2000 \times g$ for $10 \mathrm{~min}$ and, via gentle aspiration, the plasma was removed and the buffy coat discarded. The cells were washed once via centrifugation-aspiration in an isotonic PBS $\left(0.03 \mathrm{M} \mathrm{KH}_{2} \mathrm{PO}_{4}+\mathrm{Na}_{2} \mathrm{HPO}_{4} ; 290 \mathrm{mosm} / \mathrm{kg} ; \mathrm{pH}\right.$ 7.42 at $25^{\circ} \mathrm{C}$ ). Following this wash, four different RBC suspensions (hematocrit, $45 \%$ ) were prepared. 1) RBC were resuspended 

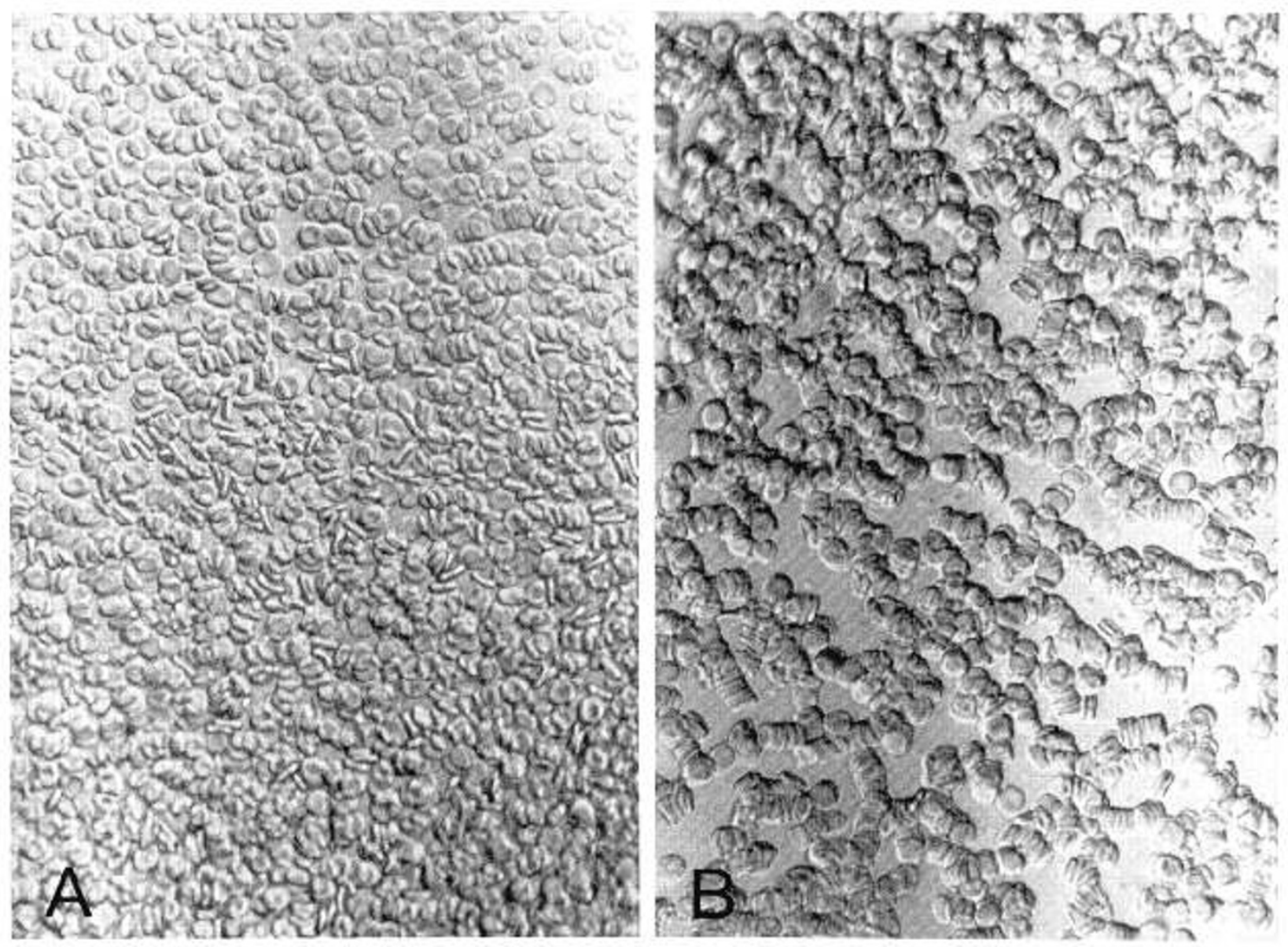

Fig. 1. Microscopic observation of RBC aggregate formation after $1 \mathrm{~min}$ of blood stasis in the rheoscope. RBC were suspended in autologous plasma. Left panel: blood with little aggregation from a preterm infant; right panel: strongly aggregating blood from an adult.

in their original plasma. 2) $\mathrm{RBC}$ from one of the adult donors (blood type $\mathrm{O}^{+}$) were resuspended in each plasma sample from both the neonates and the adults in order to evaluate the specific role of adult versus fetal plasma proteins in the aggregation process. 3) RBC from each donor were resuspended in a strongly aggregating (23) $1 \%$ dextran-PBS solution (500,000 molecular weight, T-500, lot FR 13748, Pharmacia Co., Uppsala, Sweden); the $\mathrm{pH}$ and osmolality of this dextran-PBS solution were measured to be 7.42 and $291 \mathrm{mosm} / \mathrm{kg}$. These suspensions were studied in order to evaluate the specific role of $\mathrm{RBC}$ in the aggregation process. 4) $\mathrm{RBC}$ from 10 neonates were resuspended in plasma from adults with the same blood type ( $O$ or $A$, respectively).

Rate and extent of RBC aggregation were studied using the light transmission method of Schmid-Schönbein and co-workers $(22,23)$. This technique is based on the increase of light transmission through a RBC suspension which occurs when individual $\mathrm{RBC}$ aggregate into rouleaux during blood stasis. The gaps in the suspending medium which develop between the cell aggregates produce the increased light transmission (Figs. 1 and 2).

Light transmission studies were done using a counter-rotating cone-plate rheoscope system (Effenberger, Munich, Federal Republic of Germany) consisting of a transparent glass plate and a transparent, plastic $1.5^{\circ}$ cone. The cone and the plate are rotated, in opposite directions, by a Servo-controlled DC motor. The variation of their rotational speed allows the shear rate in the 30$\mu \mathrm{m}$ gap between the cone and the plate to be changed. The rheoscope is mounted on the stage of an inverted microscope (Leitz Diavert, Leitz, Wetzlar, Germany) and the optical path is illuminated, at a wavelength of $600 \mathrm{~nm}$, by a low voltage bulb powered by a stabilized DC power supply. The light source of the microscope passes through the cone-plate portion of the rheoscope to a photocell which replaces the usual eyepiece. The

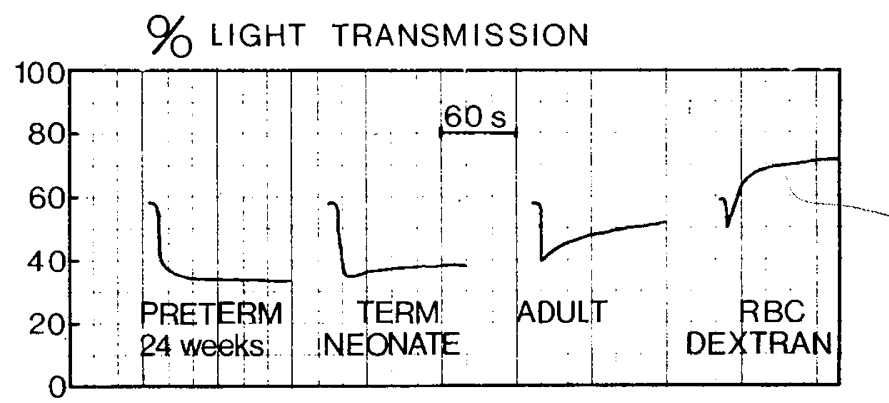

Fig. 2. Change in light transmission in the rheoscope after sudden stop of the motor. $0 \%$ indicates no light transmission (i.e. light source switched off); $100 \%$ indicates maximum light transmission (i.e. without sample). $\mathrm{RBC}$ from a preterm infant, a term neonate, and an adult were suspended in autologous plasma. RBC from the preterm infant suspended in dextran are also shown. Note the initial drop of light transmission. In the preterm infant, there was no RBC aggregation during $100 \mathrm{~s}$ of blood stasis. The term infant showed little RBC aggregation. In dextran, $\mathrm{RBC}$ aggregation was very strong.

photocell voltage is amplified and recorded on a strip chart recorder (Linear Instruments Co., Irvine, CA).

After placing $20 \mu \mathrm{l}$ of the cell suspension in the gap, the suspension is sheared at $460 \mathrm{~s}^{-1}$ for $30 \mathrm{~s}$ in order to disperse all $\mathrm{RBC}$ aggregates. The drive motor is then instantly stopped and the light transmission abruptly drops due to a transient state of random cellular orientation (Fig. 2). Following this rapid drop, the transmission increases with the time at a rate proportional to the rate of $\mathrm{RBC}$ aggregation (i.e. at a rate proportional to the formation of cell-free liquid gaps between aggregates). In cases of strong $\mathrm{RBC}$ aggregation, a steep initial rise in light transmis- 
sion is followed by a slow increase. In cases of poor RBC aggregation, the initial steep rise is missing (Fig. 2).

In order to obtain indices of the rate and final extent of RBC aggregation, two evaluation methods were used (22). 1) The initial steep rise in light transmission was recorded using a relatively rapid paper speed of $40 \mathrm{~cm} / \mathrm{min}$. This portion of the curve was differentiated and the resulting data were plotted semilogarithmically as $\ln (d V / d t)$ versus time $(t)$. The slope of the resulting straight line was obtained by least squares linear regression (3) and the $\mathrm{AHT}_{1}$ (in s) was calculated from this slope as: $\mathrm{AHT}_{1}=-\ln 2 /$ slope. Thus, $\mathrm{AHT}_{1}$ describes the initial steep rise in RBC aggregation immediately after the beginning of blood stasis. Note that AHT, cannot be determined in cases with poor $\mathrm{RBC}$ aggregation (Fig. 2). In samples with sufficient RBC aggregation, $\mathrm{AHT}_{1}$ was determined in triplicate for each suspension and the mean value of these three measurements was used for each suspension; the coefficient of variation of these individual measurements was less than $10 \%$.

2) Light transmission was recorded for 10 min using a slow paper speed of $4 \mathrm{~cm} / \mathrm{min}$ so that both the overall rate and the

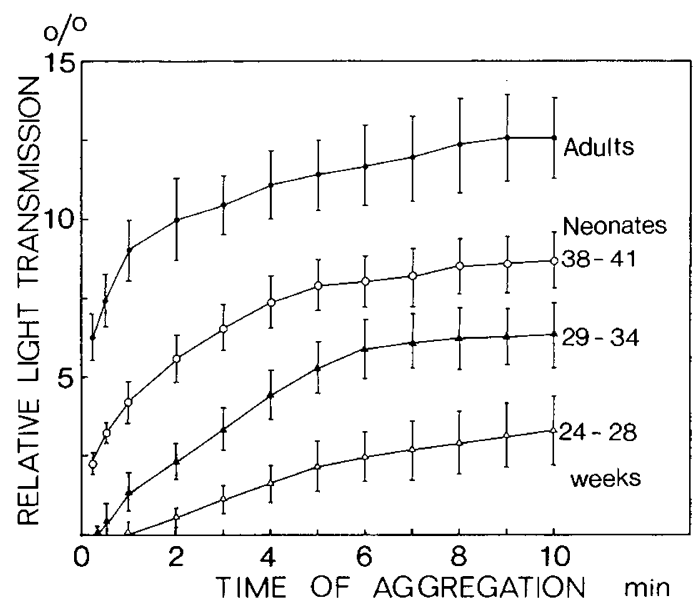

Fig. 3. Increase in relative light transmission in the rheoscope during 10 min after sudden stop of the motor. RBC were suspended in autologous plasma. Note that the percentages indicate the increase in light transmission (i.e. difference between minimum and subsequent light transmission through the sample). final extent of RBC aggregation could be assessed. In these studies, 0 and $100 \%$ light transmission (i.e. minimum light transmission with light source switched off and maximum light transmission without sample) were calibrated, as shown in Fig. 2. RBC aggregation was expressed as increase in RLT (i.e. difference between the minimum and subsequent light transmission through the sample). The maximum increase in RLT was assumed to occur after 10 min of stasis and was used to estimate the final extent of RBC aggregation (Fig. 3). The time needed to reach $50 \%$ of the maximum increase in RLT was taken to indicate the overall rate of aggregation $\left(\mathrm{AHT}_{2}\right)$.

Hence, four parameters were used to describe $\mathrm{RBC}$ aggregation (Table 1): 1) $\mathrm{AHT}_{1}$ is derived from the initial rapid rise in $\mathrm{RBC}$ aggregation immediately after the beginning of blood stasis. It is in the order of a few seconds and describes the rate of initial aggregation. 2) $\mathrm{AHT}_{2}$ is derived from 10-min recordings of light transmission and describes the overall rate of $\mathrm{RBC}$ aggregation. 3) Increase in RLT is measured during 1 min of stasis. In adults, $\mathrm{RBC}$ aggregation nearly reaches the maximum after $1 \mathrm{~min}$ of stasis (Fig. 3). 4) Increase in RLT is measured during $10 \mathrm{~min}$ of stasis. It indicates the maximum or final extent of $\mathrm{RBC}$ aggregation.

The hematocrits of $\mathrm{RBC}$ suspensions were determined using the microhematocrit technique. The values were not corrected for trapped plasma which is about $2 \%$ in full term neonates and adults (11). Solution osmolalities were measured by freezing point depression (Model 2007, Precision Systems, Sudbury, MA) and $\mathrm{pH}$ by a Radiometer Model PHM 71 system (Radiometer Co., Copenhagen, Denmark) operating at $25^{\circ} \mathrm{C}$. Plasma fibrinogen concentrations were measured via a radial immunodiffusion technique (M-Partigen Fibrinogen Kit, Calbiochem-Behring Co., La Jolla, CA).

Analysis of variance was used to test for differences in measurements among the two groups of preterm infants, the full term neonates, and the adults (3). Regression analyses were utilized to determine overall correlations between fibrinogen and increase in relative light transmission.

\section{RESULTS}

The plasma fibrinogen levels and the aggregation results for the 25 neonates and the 13 adults are summarized in Table 1. Figure 3 shows the rate of aggregation measured as an increase in light transmission in the rheoscope during $10 \mathrm{~min}$ of stasis (i.e. after sudden stoppage of the rheoscope motor). Examination

Table 1. Aggregation data for blood from preterm and full term neonates and adults*

\begin{tabular}{|c|c|c|c|c|c|}
\hline & \multicolumn{3}{|c|}{ Neonates (wk of gestation) } & $\begin{array}{c}\text { Adults } \\
\text { (d) } \\
n=13\end{array}$ & $\begin{array}{l}\text { Significant } \\
\text { differences among } \\
\text { the four groups } \\
(p<0.05)\end{array}$ \\
\hline Fibrinogen $(\mathrm{mg} / \mathrm{dl})$ & $181 \pm 85$ & $262 \pm 69$ & $245 \pm 63$ & $354 \pm 48$ & $\mathrm{a}<\mathrm{b}=\mathrm{c}<\mathrm{d}$ \\
\hline \multicolumn{6}{|l|}{$\mathrm{RBC}$ in autologous plasma } \\
\hline RLT after $1 \min (\%)$ & $0.3 \pm 0.5$ & $1.4 \pm 1.6$ & $4.2 \pm 1.8$ & $9.1 \pm 3.3$ & $\mathrm{a}=\mathrm{b}<\mathrm{c}<\mathrm{d}$ \\
\hline RLT after $10 \min (\%)$ & $3.4 \pm 2.4$ & $6.4 \pm 3.2$ & $8.6 \pm 2.6$ & $12.5 \pm 4.6$ & $\mathrm{a}<\mathrm{c}<\mathrm{d} ; \mathrm{a}=\mathrm{b}<\mathrm{d}$ \\
\hline \multicolumn{6}{|c|}{$\begin{array}{l}\text { Adult } \mathrm{RBC} \text { in neonatal and adult } \\
\text { plasma }\end{array}$} \\
\hline $\mathrm{AHT}_{1}(\mathrm{~s})$ & NM & NM & $2.95 \pm 0.62$ & $1.52 \pm 0.41$ & $c>d$ \\
\hline $\mathrm{AHT}_{2}(\mathrm{~s})$ & $271 \pm 97$ & $181 \pm 80$ & $92 \pm 33$ & $20 \pm 11$ & $a>b>c>d$ \\
\hline RLT after $1 \min (\%)$ & $16.9 \pm 3.9$ & $18.8 \pm 5.6$ & $19.8 \pm 6.6$ & $20.2 \pm 7.6$ & $a=b=c=d$ \\
\hline RLT after $10 \min (\%)$ & $20.8 \pm 5.3$ & $25.9 \pm 7.3$ & $25.0 \pm 7.1$ & $24.7 \pm 8.7$ & $a=b=c=d$ \\
\hline
\end{tabular}

* All values are mean $\pm \mathrm{SD}$. NM, not measurable in most infants of this group. 
of these results leads to the following observations. 1) The fibrinogen levels were significantly decreased in the whole group of neonates when compared to the adults. The smallest infants showed the lowest fibrinogen levels. 2) The aggregation half-time derived from the initial rapid increase in light transmission $\left(\mathrm{AHT}_{1}\right)$ was significantly longer in the full term infants when compared to the adults. In the preterm infants, the slope was too flat for this evaluation (Fig. 2). 3) The time-dependent increase in light transmission during $10 \mathrm{~min}$ of stasis demonstrated markedly different RBC aggregation patterns (Fig. 3); after 1 min of stasis, there was almost no RBC aggregation in the most immature infants, little aggregation in the infants with 29 to 34 wk of gestation, marked aggregation in the full term neonates, and strong RBC aggregation in the adults. After 10 min of stasis, aggregation was evident in all infants but was clearly dependent on the gestational age of the infants as was the time needed to reach $50 \%$ of the $10-\mathrm{min}$ value. 4 ) For the combined data from the 25 neonates and the 13 adults (Fig. 4), there were significant exponential relationships between fibrinogen concentration and the aggregation data.

Table 1 also presents aggregation results for $\mathrm{RBC}$ from one adult donor resuspended in the neonatal and adult plasma samples. There were no significant differences between the neonatal and the adult $\mathrm{RBC}$ when suspended in the plasma from the neonates. Thus, the origin of the RBC was without effect on the aggregating properties of the plasma samples.

The results of the RBC-dextran suspensions (Table 1) show that the $\mathrm{RBC}$ from all four groups aggregated equally in this medium. In 10 neonates, RBC were also resuspended in adult plasma samples with compatible blood types. These suspensions also show no significant differences between neonatal and adult RBC (Fig. 5). Thus, different plasma properties, rather than different RBC properties, appear to be responsible for the decreased $R B C$ aggregation noted in the neonates' plasma-RBC suspensions.

\section{DISCUSSION}

From the present data, we come to three major conclusions. 1) Both the speed and final extent of RBC aggregation are markedly lower in small preterm infants than in full term neonates and adults. 2) The weak RBC aggregation in small preterm infants is associated with low fibrinogen levels. 3) Exchange of the original plasma for adult plasma or for strongly aggregating dextran increases RBC aggregation in preterm infants to adult values.

\section{Aggregation after $1 \mathrm{~min}$ of stasis}

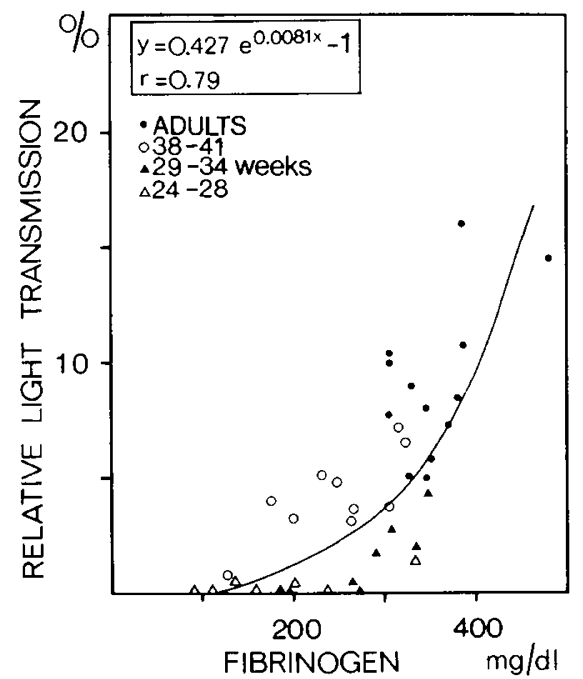

In general, decreased $\mathrm{RBC}$ aggregation in the neonates correlated with lower fibrinogen levels (Fig. 4). However, the preterm infants tended to have weaker RBC aggregation than the full term infants at similar fibrinogen levels. This was particularly evident after $1 \mathrm{~min}$ of stasis where RBC aggregation in the preterm infants approached zero at fibrinogen levels below 250 $\mathrm{mg} / \mathrm{dl}$ while term neonates with similar fibrinogen levels still showed RBC aggregation (Fig. 4). Thus, other factors appear to contribute to the weak RBC aggregation in preterm infants. In addition to fibrinogen, several other plasma proteins such as immunoglobulins, albumin, transferrin, and several clotting factors are lower in small preterm infants than in term neonates $(2$, $6,9,10,17,21)$. Immunoglobulins, in particular, promote RBC
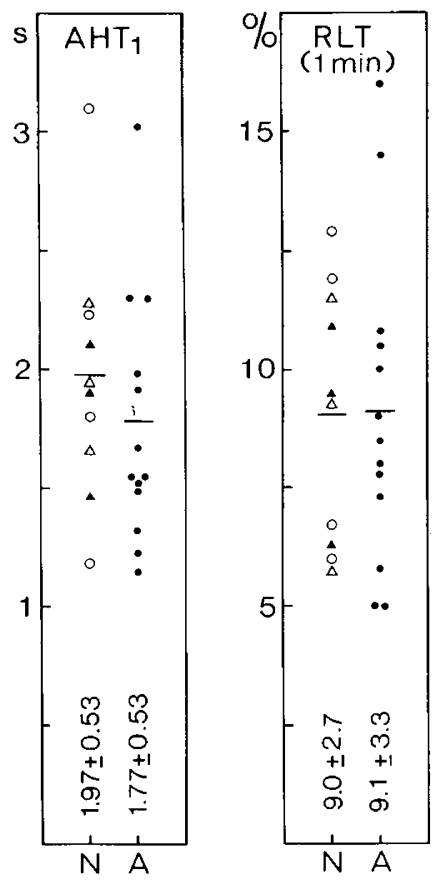

Fig. 5. Aggregation of neonatal RBC in adult plasma. Left panel: $\mathrm{AHT}$, derived from the initial rapid increase in light transmission; right panel: increase in RLT after $1 \mathrm{~min}$ of blood stasis. RBC from 10 neonates $(N)$ were suspended in plasma from adults. RBC from 13 adults $(A)$ suspended in autologous plasma are also shown. Symbols are as in Figures 3 and 4 .

Aggregation after $10 \mathrm{~min}$ of stasis

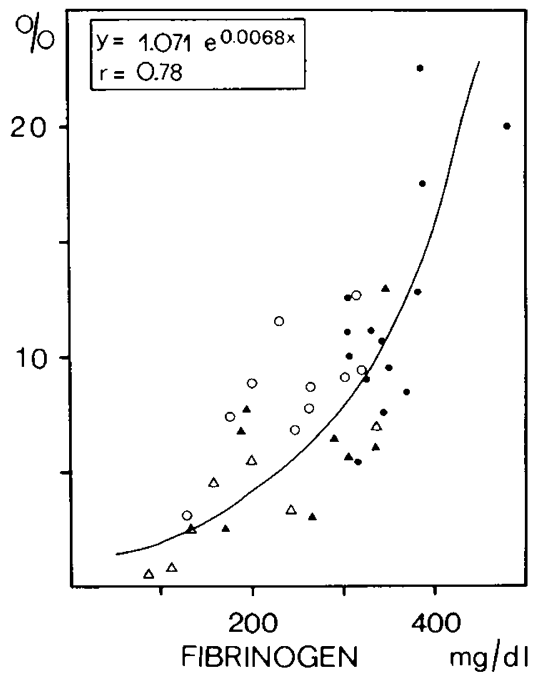

Fig. 4. Increase in relative light transmission after 1 and $10 \mathrm{~min}$ of blood stasis is plotted against fibrinogen concentration (exponential regressions). RBC were suspended in autologous plasma. 
aggregation because of their relatively large size although their effect is markedly less than that of fibrinogen (4). Whether a special type of fetal fibrinogen (26) causes decreased RBC aggregation is unknown.

The aggregation tendency of $\mathrm{RBC}$ also depends on cellular properties such as the electrical charge of cell surface, $\mathrm{RBC}$ deformability, and cell geometry (4). Electrical charge (16), deformability (14), and shape (15) of neonatal and adult RBC are not different. This concurs with our finding that $\mathrm{RBC}$ from all donors showed similar aggregation when suspended in dextran or in plasma from adult donors (Table 1, Fig. 5). Thus, the specific properties of neonatal RBC (e.g. size, hemoglobin F) do not appear to affect aggregation.

An important effect of RBC aggregation is the steep increase in blood viscosity at low shear rates (4). Our aggregation data explain why this rise in blood viscosity with decreasing shear rate is markedly less in small preterm infants than in term neonates and adults (13). Decreased RBC aggregation also explains the low erythrocyte sedimentation rate in newborn infants (1).

The most striking conclusion from this study is that in most preterm infants with less than 29 weeks of gestation there is no $\mathrm{RBC}$ aggregation even after 1 min of stasis, whereas in term neonates and adults, RBC aggregates are formed within a few seconds of blood stasis. Implications are, however, at this point highly speculative. One of these implications could be that small preterm infants have a low risk of thromboembolic accidents. Another speculation is that cardiac arrest lasting $1 \mathrm{~min}$ will not cause noticeable $\mathrm{RBC}$ aggregation in small preterm infants, whereas in term neonates and adults, it would cause RBC aggregation in the entire circulation. It is unknown whether cardiac massage initiated after a short period of cardiac arrest is more successful if RBC aggregation is slow and weak because smaller shear forces are required to redisperse $\mathrm{RBC}$ aggregates.

Under physiological conditions in vivo, RBC aggregation takes place only in veins where shear conditions are low (5). Thus, decreased $\mathrm{RBC}$ aggregation in preterm infants implies that a lower pressure gradient is required to drive blood through their veins. This may contribute to the peculiar circulation of preterm infants, who characteristically have low vascular pressures, low vascular resistances and high flow conditions $(12,25,27)$.

Besides these favorable hemorheological properties in preterm infants, there may be negative aspects as well. Because RBC aggregation plays a role in coagulation (18), weak RBC aggregation might contribute to the tendency of small preterm infants to develop intracranial hemorrhage. This speculation is supported by the finding of low plasma fibrinogen in many preterm infants with intracranial hemorrhage (17). On the other hand, an increase in plasma fibrinogen and RBC aggregation might require raised arterial-capillary-venous driving pressures to maintain the microcirculation, thereby increasing the risk of cerebral vessel rupture.

We conclude from our data that RBC aggregation is markedly decreased in small preterm infants at birth when compared to term neonates and adults. We speculate that the weak RBC aggregation in preterm infants might play a role in maintaining the peculiarities of their circulation but also in their tendency to develop intracranial hemorrhage. An increase in fibrinogen and/ or other plasma proteins may increase $\mathrm{RBC}$ aggregation, thereby altering the characteristic blood flow conditions in preterm infants. Serial (prospective) hemorheological, circulatory, and coagulation studies on preterm infants are necessary to evaluate the role of $\mathrm{RBC}$ aggregation in postnatal complications.
Acknowledgments. The authors wish to thank Rosalinda Wenby, B. S., for her technical assistance and Vivian M. Vargas, M.S.B.A., for processing the films and prints.

\section{REFERENCES}

1. Adler SM, Denton RL 1975 The erythrocyte sedimentation rate in the newborn period. J Pediatr 86:942

2. Barnard DR, Simmons MA, Hathaway WE 1979 Coagulation studies in extremely premature infants. Pediatr Res 13:1330

3. Campbell RC 1967 Statistics for Biologists. Cambridge University Press, London

4. Chien S 1972 Present state of blood rheology. In: Messmer K, SchmidSchönbein $\mathrm{H}$ (eds) Hemodilution. Theoretical Basis and Clinical Application. Karger, Basel, pp I-45

5. Cokelet GR 1980 Dynamics of red blood cell deformation and aggregation, and in vivo flow. In Cokelet GR, Meiselman HJ, Brooks DE (eds) Erythrocyte Mechanics and Blood Flow. Alan R Liss, New York, pp 141-148

6. Foley ME, Isherwood DM, McNicol GP 1978 Viscosity, haematocrit, fibrinogen and plasma proteins in maternal and cord blood. Br J Obstet Gynecol $85: 500$

7. Fukada E, Kaibara M 1980 Viscoelastic study of aggregation of red blood cells. Biorheology 17:177

8. Gaehtgens P. Schickendantz S 1975 Rheologic properties of maternal and neonatat blood. Bibl Anat 13:107

9. Hathaway WE 1975 The bleeding newborn. Semin Hematol 12:175

10. Jürgens H, Göbel U, Bokelmann J, Voss H, Wahn V 1979 Coagulation studies on umbilical arterial and venous blood from normal newborn infants. Eur $\mathbf{J}$ Pediatr 131:199

11. Linderkamp O, Meiselman HJ, Wu PYK, Miller FC 1981 Blood and plasma viscosity and optimal hematocrit in the normal newborn infant. Clin Hemorheol 1:575

12. Linderkamp O, Strohhacker I, Versmold HT, Klose H, Riegel KP, Betke K 1979 Peripheral circulation in the newborn. Interaction of peripheral blood flow, biood pressure. blood volume, and blood viscosity. Eur J Pediatr 129:73

13. Linderkamp O, Versmold HT, Riegel KP, Betke K 1984 Contributions of red cells and plasma to blood viscosity in preterm and full-term infants and adults. Pediatrics 74:45

14. Linderkamp O, Wu PYK, Meiselman HJ 1982 Deformability of density separated red blood cells in normal newborn infants and adults. Pediatr Res 16:964

15. Linderkamp O, Wu PYK, Meiselman HJ 1983 Geometry of neonatal and adult red blood cells. Pediatr Res 17:250

16. Luner SJ, Szklarek D 1975 Comparison of electrophoretic mobility and membrane sialic acid content of erythrocytes from adult and umbilical cord blood. Pediatr Res 9:583

17. McDonald MM, Johnson ML, Rumack CM, Guggenheim MA, Koops BL Hathaway WE 1982 The role of coagulopathy in neonatal intracranial hemorrhage (ICH). Pediatr Res 16:209A (abstr)

18. Meiselman HJ, Goldsmith HL 1973 Blood rheology, blood flow and thrombosis. Thromb Diath Haemorrh 54 (suppl):273

19. Oseas RS, Toloza EM, Miller ME 1982 Cord blood polymorphonuclear leucocyte aggregation. Pediatr Res 16:210A

20. Ozanne P. Linderkamp O, Miller FC, Meiselman HJ 1983 Erythrocyte aggregation during normal pregnancy. Am J Obstet Gynecol 147:576

21. Pilgrim U, Fontanellaz HP, Evers G, Hitzig WH 1975 Normal values of immunoglobulins in premature and full-term infants, calculated as percentiles. Helv Paediatr Acta 30:121

22. Schmid-Schönbein H, Kline KA, Heinich L, Volger E, Fischer $\Upsilon 1975 \mathrm{Mi}-$ crorheology and light transmission of blood. III. The velocity of red cell aggregation formation. Pflügers Arch 354:299

23. Schmid-Schönbein H, Volger E, Klose HJ 1972 Microrheology and light transmission of blood. Il. The photometric quantification of red cell aggregate formation and dispersion in flow. Pflügers Arch 333:140

24. Schmid-Schönbein H, Weiss J, Ludwig H 1973 A simple method for measuring red cell deformability in models of the microcirculation. Blut 26:369

25. Versmold HT, Kittermann JA. Phibbs RH, Gregory GA, Tooley WH 1981 Aortic blood pressure during the first 12 hours of life in infants with birth weight 610 to 4,220 grams. Pediatrics 67:607

26. Witt I, Müller H, Künzer W 1969 Evidence for the existence of foetal fibrinogen. Thromb Diath Haemorrh 22:101

27. Wu PYK, Wong WH, Guerra G, Miranda R, Godoy RR, Preston B, Schoentgen S, Levan NE 1980 Peripheral blood flow in the neonate. 1. Changes in total, skin, and muscle blood flow with gestational age and postnatal age. Pediatr Res 14:1374 